\title{
ENTERITIS IN JUVENILE SYSTEMIC ERITEMATOSUS LUPUS
}

Victor da Silva Coelho (Universidade Federal de Juiz de Fora , Juiz de Fora, MG, Brasil), Diana Campos Fernandino (Universidade Federal de Juiz de Fora, Juiz de Fora, MG, Brasil), Paula Reale Fernandes

(Universidade Federal de Juiz de Fora, Juiz de Fora, MG, Brasil), Ludmila Godinho Figueiredo (Universidade Federal de Juiz de Fora, Juiz de Fora, MG, Brasil), Vania Schinzel (Universidade Federal de Juiz de Fora , Juiz de Fora, MG, Brasil)

\section{BACKGROUND}

Lupus is a multisystemic autoimmune disease that affects connective tissue. Gastrointestinal manifestation is common either by the disease itself, with autoimmune hepatitis or pancreatitis, or by side effects of medications. In this context, enteritis, secondary to small vessel vasculitis, is a severe and rare complication, with an incidence of $0.2 \%$ in adult patients. Its prevalence in pediatric patients is unknown and there are few available studies on its management in this population. We describe our experience with this potentially fatal manifestation.

\section{CASE REPORT}

YSP, 13 years old, female, in December/2018 presented with polyarthritis in hands, wrists, knees, ankles, cervical and lumbar spine, associated with joint stiffness, morphea on back and $5 \mathrm{~kg}$ weight loss. No other complaints. Exams revealed FR(-), FAN 1: 320 NPFD, ENA (-), complement consumption, increased inflammatory tests, negative viral serologies and no radiographic changes. We started treatment with methotrexate and prednisone for Juvenile Idiopathic Arthritis Polyarticular FR (-). There was progressive improvement. In April /2019, she reported fever for 1 week, associated with alopecia, malar rash, painless oral ulcers on the palate, adenomegaly in the anterior cervical chain and inguinal chain, precordial pain, and upper abdominal pain, lymphopenia, thrombocytopenia, and weight loss. She did not present with vomiting or diarrhea in the period. New laboratory tests with FAN 1: $640 \mathrm{NH}$, ENA (-), anti-DNA (+), consumption of complement. Confirmed diagnosis of SLE. The patient was hospitalized and performed an acute abdomen procedure, and pancreatitis was ruled out. Computed tomography (CT) of the abdomen was performed, showing parietal edema with mucous enhancement of the cecum and ascending colon, with a slight densification of adjacent fat, suggesting an inflammatory process; lymph node enlargement in retroperitoneal, mesenteric, pelvic and inguinal chains; and free fluid in the peritoneal cavity. The echocardiogram showed mild pericardial effusion without hemodynamic repercussion. Antimicrobial treatment was instituted and, after 48 hours, pulsetherapy with methylprednisolone $(1 \mathrm{~g} / \mathrm{day}, 3$ days). The patient improved completely from the intestinal tract without the need for surgical intervention. She is being treated with monthly pulse therapy with methylprednisolone, oral use of prednisone, azathioprine, methotrexate and hydroxychloroquine, without further complications.

\section{CONCLUSION}

Enteritis is a severe manifestation of SLE, often underdiagnosed. Usually presents with abdominal pain and vomiting, bowel obstruction and even necrosis. Early diagnosis is critical to avoid progression to fatal outcomes. More studies are needed to establish the best therapy for these cases. 